\title{
Radio wave emissions due to gravitational radiation
}

\author{
Mattias Marklund ${ }^{1,3}$, Gert Brodin ${ }^{2,4}$, and Peter K. S. Dunsby ${ }^{1,5}$
}

\begin{abstract}
We consider the interaction of a weak gravitational wave with electromagnetic fields in a thin plasma on a Minkowski background spacetime using the $1+3$ orthonormal frame formalism. Because gravitational and electromagnetic waves satisfy the same dispersion relation, electromagnetic waves can be effectively generated as a result of this interaction. In the case of the interaction with a static magnetic field, the amplitude of the electromagnetic waves depends on the size of the excitation region in which the magnetic field is contained. It is argued that due to the presence of a plasma this process can also lead to the generation of higher harmonics of the original mode. Estimates are given for this effect in the case of a binary pulsar and a cold electron plasma. It is found that the emmited radiation will lie in the radio frequency band. We also speculate on the possible relevance of this process on situations in cosmology, in particular whether this could be used to constrain primordial magnetic fields.
\end{abstract}

Subject headings: Gravitation — plasmas — pulsars: general — radiation mechanisms: non-thermal — radio continuum: general — relativity

\section{Introduction}

It is well known that electromagnetic (EM) waves can scatter off gravitational fields. For instance, DeWitt \& Brehme (1960) considered raditation damping in a gravitational field, and Stephani (1982) gives a general discussion on the subject. One can distinguish between two main areas:

(a) The analysis of effects due to time-independent gravitational fields, such as Schwarzschild or Kerr spacetime, in an astrophysical context (Macdonald \& Thorne 1982; Daniel \& Tajima 1997), or

(b) scattering of EM fields off time-dependent gravitational fields (Cooperstock 1968; Zeldovich 1973; Gerlach 1974; Denisov 1978; Grischuk \& Polnarev 1980; Demiański 1985).

\footnotetext{
${ }^{1}$ Department of Mathematics and Applied Mathematics, University of Cape Town, Rondebosch 7701, South Africa

${ }^{2}$ Department of Plasma Physics, Umeå University, S-901 87 Umeå, Sweden

${ }^{3}$ E-mail address: marklund@shiva.mth.uct.ac.za

${ }^{4}$ E-mail address: gert.brodin@physics.umu.se

${ }^{5}$ E-mail address: peter@vishnu.mth.uct.ac.za
} 
The latter studies (b) have shown that there can be a coherent interaction between linear gravitational waves and EM fields, which would provide an effective means of transfering energy from gravitaional to EM degrees of freedom. It is for this reason that subsequent work focused on using this mechanism, as a way of detecting gravitational waves (Lupanov 1967; Braginskii et al. 1973; Grishchuk \& Sazhin 1975).

Motivated by the discussion given in the references above, we return to the time-dependent problem in order to further investigate a number of effects which arise when a gravitational wave interacts with an EM field, this time with a plasma present. In this letter we focus on a simple example, in order to demonstrate the basic effect, and in order to maximise clarity and make physical interpretations simple, we will use the $1+3$ orthonormal frame (ONF) formalism (see, e.g., Ellis \& van Elst (1998) and references therein). This has the advantage of picking out a physical observer and splitting spacetime relative to her.

The paper is organized as follows: In section 2 we briefly review the ONF formalism and rewrite Maxwells equations using our ONF, introducing effective (gravity induced) charge and current densities. Next, in section 3, we specify the metric to that of a weak one-dimesional gravitational wave, and calculate the correpsonding (effective) current denstites. Section 4 then considers the excitation of an electromagnetic wave by a gravitational wave in a magnetized thin one-component plasma. We deduce that the resulting electromagnetic field amplitude is proportional to the gravitational wave amplitude, the magnitude of the static magnetic field and the size of the interaction region. In section 5 we consider the interaction region exterior to a binary pulsar. This consists of a vacuum regime (swept out by the Poynting flux) and a regime of interstellar matter. In order to simplify our analysis we consider only regions in which the magnetic field is on average aligned with the axis of rotation of the binary pulsar. Making an analog to laboratory experiments we argue that efficient harmonic generation of the EM waves may take place, and rough estimates suggest that these harmonics might - for favorable choices of parameters - be observable by the proposed Astronomical Low Frequency Array (ALFA) (Jones et al. 1998). Finally, our results are summarized and discussed in section 6 .

\section{Preliminaries}

Suppose an observer moves with 4 -velocity $u^{a}(a=0, \ldots, 3)$, where $u_{a} u^{a}=1$ (we put $c=1$ ). This observer will measure the electric and magnetic fields (Ellis \& van Elst 1998)

$$
E_{a} \equiv F_{a b} u^{b}, \quad B_{a} \equiv \frac{1}{2} \epsilon_{a b c} F^{b c},
$$

respectively, where $F_{a b}$ is the EM field tensor. Here $\epsilon_{a b c} \equiv u^{d} \eta_{d a b c}$, where $\eta_{a b c d}$ is the totally skew 4-dimensional volume element, and $\eta_{0123}=\left|\operatorname{det}\left(g_{a b}\right)\right|^{1 / 2}$ (which will be just equal to 1 in our ONF case). With these definitions, we can write the field tensor as

$$
F^{a b}=u^{a} E^{b}-u^{b} E^{a}+\epsilon^{a b c} B_{c} .
$$


We now introduce the ONF: $e_{a}, a=0, \ldots, 4$, where $e_{0}=u$, so that, with the above splitting (2) of the field tensor, Maxwell's equations $\nabla_{b} F^{a b}=\mu_{0} j^{a}, \nabla_{[a} F_{b c]}=0 \mathrm{read}$

$$
\begin{aligned}
\boldsymbol{\nabla} \cdot \boldsymbol{E} & =\rho_{E}+\rho_{\mathrm{m}} / \varepsilon_{0}, \\
\boldsymbol{\nabla} \cdot \boldsymbol{B} & =\rho_{B}, \\
e_{0}(\boldsymbol{E})-\nabla \times \boldsymbol{B} & =-\boldsymbol{j}_{E}-\mu_{0} \boldsymbol{j}_{\mathrm{m}}, \\
e_{0}(\boldsymbol{B})+\nabla \times \boldsymbol{E} & =-\boldsymbol{j}_{B},
\end{aligned}
$$

where the "effective" (gravity induced) charge densities and current densities are given by

$$
\begin{aligned}
\rho_{E} & \equiv-\Gamma_{\beta \alpha}^{\alpha} E^{\beta}-\epsilon^{\alpha \beta \gamma} \Gamma_{\alpha \beta}^{0} B_{\gamma}, \\
\rho_{B} & \equiv-\Gamma_{\beta \alpha}^{\alpha} B^{\beta}+\epsilon^{\alpha \beta \gamma} \Gamma_{\alpha \beta}^{0} E_{\gamma}, \\
j_{E} & \equiv\left[-\left(\Gamma_{0 \beta}^{\alpha}-\Gamma_{\beta 0}^{\alpha}\right) E^{\beta}+\Gamma_{0 \beta}^{\beta} E^{\alpha}-\epsilon^{\alpha \beta \gamma}\left(\Gamma_{\beta 0}^{0} B_{\gamma}+\Gamma_{\beta \gamma}^{\delta} B_{\delta}\right)\right] e_{\alpha}, \\
j_{B} & \equiv\left[-\left(\Gamma_{0 \beta}^{\alpha}-\Gamma_{\beta 0}^{\alpha}\right) B^{\beta}+\Gamma_{0 \beta}^{\beta} B^{\alpha}+\epsilon^{\alpha \beta \gamma}\left(\Gamma_{\beta 0}^{0} E_{\gamma}+\Gamma_{\beta \gamma}^{\delta} E_{\delta}\right)\right] e_{\alpha},
\end{aligned}
$$

while $\rho_{\mathrm{m}}$ and $\boldsymbol{j}_{\mathrm{m}}$ are the matter charge and current densities, respectively. Here $\Gamma_{b c}^{a}$ are the Ricci rotation coefficients, and we have introduced the three-vector notation $\boldsymbol{E} \equiv\left(E^{\alpha}\right)=\left(E^{1}, E^{2}, E^{3}\right)$ etc., and $\boldsymbol{\nabla} \equiv\left(e_{1}, e_{2}, e_{3}\right)$. The dot - and cross - products are defined in the usual Euclidian way.

\section{EM wave excitation by weak gravitational radiation}

To first order, the solution to Einstein's vacuum equations can be written as (using the transverse traceless gauge):

$$
\mathrm{d} s^{2}=-\mathrm{d} t^{2}+(1+h) \mathrm{d} x^{2}+(1-h) \mathrm{d} y^{2}+\mathrm{d} z^{2},
$$

where $h$ is the deviation from flat space satisfying $|h| \ll 1$, and we consider only the +- polarisation of the gravitational wave. The deviation $h$ is a solution to the wave equation $\square h=0$, i.e. $h=h(z-t)$ (Misner, Thorne, \& Wheeler 1973). In order to clarify the physical intepretation and to simplify the equations, we introduce a contravariant $\mathrm{ONF}$

$$
e_{0}=\partial_{t}, e_{1}=\left(1-\frac{1}{2} h\right) \partial_{x}, e_{2}=\left(1+\frac{1}{2} h\right) \partial_{y}, e_{3}=\partial_{z},
$$

such that the metric components become $\eta_{a b}=\operatorname{diag}(-1,1,1,1)$. Thus the local rest - space orthogonal to a synchronus observer takes Euclidian form.

For the gravitational wave (8), the charge densities (4) and (5) are zero. The current densities (6) and (7) have components

$$
\begin{aligned}
& j_{E}^{1}=-\frac{1}{2}\left(-E^{1}+B^{2}\right) \dot{h}=j_{B}^{2} \\
& j_{E}^{2}=-\frac{1}{2}\left(E^{2}+B^{1}\right) \dot{h}=-j_{B}^{1}
\end{aligned}
$$

where $\dot{h}=\partial h / \partial z=-\partial h / \partial t$. 


\section{1 - D example}

Here we consider the effects of an incoming gravitational wave on a static magnetic field in an inhomogeneous plasma, restricting our attention to a 1 - D case. Thus, we assume the presence of a external static magnetic field $\widehat{\boldsymbol{B}}=\widehat{B}(z) e_{1}$, and study gravitational wave excitation of small amplitude plasma waves propagating in the $z$-direction. We take the gravitational wave to be monochromatic, i.e. $h=\bar{h} \exp [\mathrm{i} k(z-t)]$, and we let the frequency of the excited wave coincide with the driver, that is, $E^{a}=\bar{E}^{a}(z) \exp (-\mathrm{i} \omega t)$, where $\omega=k$, and similarly for all other quantities. From Maxwells equations (3), using $|\bar{B}(z)|,|\bar{E}(z)| \ll|\widehat{B}(z)|$ and equations (10), we immediately obtain ${ }^{6}$

$$
\begin{aligned}
\left(\frac{\partial^{2}}{\partial z^{2}}+k^{2}\right) \bar{E}^{2}+\mathrm{i} \omega \mu_{0} \bar{j}_{\mathrm{m}}^{2} & =-\left(k^{2} \widehat{B}-\frac{1}{2} \mathrm{i} k \frac{d \widehat{B}}{d z}\right) \bar{h} \exp (\mathrm{i} k z) \equiv S(z), \\
\omega^{2} \bar{E}^{3}+\mathrm{i} \omega \mu_{0} \bar{j}_{\mathrm{m}}^{3} & =0 .
\end{aligned}
$$

In order to calculate the current, we assume the ions to be immobile - that is $\omega \gg \omega_{\text {pi }}$, where $\omega_{\text {pi }}$ is the ion plasma frequency. Using our ONF the equation of motion for cold electrons can be written

$$
m u^{a} \boldsymbol{v}_{; a}=q\left(v^{0} \boldsymbol{E}+\boldsymbol{v} \times \boldsymbol{B}\right),
$$

where $v^{a}=\left(v^{0}, \boldsymbol{v}\right)$ is the electron four - velocity, satisfying $v^{a} v_{a}=-1$, and $u^{a}$ is the observers four velocity. Linearizing the equation of motion (13a) around the unperturbed plasma state (i.e. $\boldsymbol{E}=0$, $\boldsymbol{v}=0$ ) and dropping all cross terms containting the three-velocity and the rotation coefficients, we obtain

$$
-\mathrm{i} m \omega \overline{\boldsymbol{v}}=q(\overline{\boldsymbol{E}}+\overline{\boldsymbol{v}} \times \widehat{\boldsymbol{B}}) .
$$

Solving equation (13b) for the electron velocity we obtain

$$
\begin{aligned}
& \bar{v}^{2}=\frac{q}{m \omega}\left(\mathrm{i} \bar{E}^{2}+\frac{\omega_{\mathrm{c}}}{\omega} \bar{E}^{3}\right)\left(1-\frac{\omega_{\mathrm{c}}^{2}}{\omega^{2}}\right)^{-1}, \\
& \bar{v}^{3}=\frac{q}{m \omega}\left(\mathrm{i} \bar{E}^{3}-\frac{\omega_{\mathrm{c}}}{\omega} \bar{E}^{2}\right)\left(1-\frac{\omega_{\mathrm{c}}^{2}}{\omega^{2}}\right)^{-1},
\end{aligned}
$$

where $\omega_{\mathrm{c}}=q \widehat{B} / \mathrm{m}$ is the electron cyclotron frequency. Using $\boldsymbol{j}_{\mathrm{m}}=q n_{0} \boldsymbol{v}$ where $n_{0}$ is the unperturbed electron number density at rest, and inserting equations (14) in (11) and (12), we obtain a driven wave equation for the extra-ordinary EM wave

$$
\left(\frac{\partial^{2}}{\partial z^{2}}+k^{2}-\Delta k^{2}\right) \bar{E}^{2}=S(z),
$$

where the plasma induced wavenumber mismatch $\Delta k$ is found to be

$$
\Delta k^{2}=\frac{\omega_{\mathrm{p}}^{2}\left(\omega^{2}-\omega_{\mathrm{p}}^{2}\right)}{\omega^{2}-\omega_{\mathrm{h}}^{2}} .
$$

\footnotetext{
${ }^{6}$ We linearize such that terms quadratic in barred quantities are dropped.
} 
Here $\omega_{\mathrm{p}}=\left(n_{0} q^{2} / \varepsilon_{0} m\right)^{1 / 2}$ is the plasma frequency and $\omega_{\mathrm{h}}=\left(\omega_{\mathrm{p}}^{2}+\omega_{\mathrm{c}}^{2}\right)^{1 / 2}$ is the upper hybrid frequency. Assuming that the static field is localized in the region $0<z<a$, the exact solution to equation (15), when $\Delta k=$ constant,${ }^{7}$ is

$$
\begin{aligned}
\bar{E}^{2}(z)= & \exp \left[\mathrm{i} k_{\mathrm{loc}} z\right] \int_{0}^{z} \exp \left[-\mathrm{i} k_{\mathrm{loc}} z^{\prime}\right] S\left(z^{\prime}\right) \mathrm{d} z^{\prime} \\
& +\exp \left[-\mathrm{i} k_{\mathrm{loc}} z\right] \int_{a}^{z} \exp \left[\mathrm{i} k_{\mathrm{loc}} z^{\prime}\right] S\left(z^{\prime}\right) \mathrm{d} z^{\prime},
\end{aligned}
$$

where the integration limits have been chosen so that there are no waves coming into the excitation region. Here $k_{\text {loc }} \equiv\left(k^{2}-\Delta k^{2}\right)^{1 / 2}$ is the local wave number of the electromagnetic radiation. The solution (17) also holds approximately when both $|\Delta k| \ll|k|$ and $|d \Delta k / d z| \ll|k \Delta k|$. We note that for a large excitation region, $k a \gg 1$, EM waves propagating along the positive $z$-axis can obtain much higher amplitudes than those in the opposite direction, provided the mismatch is small, that is $\Delta k \ll k$. In the next section we will consider the regime

$$
\omega_{\mathrm{p}}^{2} / \omega_{\mathrm{c}} \ll \omega \lesssim \omega_{\mathrm{p}} \ll \omega_{\mathrm{c}}
$$

in which case the condition $\Delta k \ll k$ is fulfilled. Focusing on this approximation, we may neglect the negative propagating wave all together, and write the solution in the outgoing region $z>a$ as

$$
\bar{E}^{2}(z, t)=E_{\text {out }} \exp \left[\mathrm{i}\left(k_{\text {out }} z-\omega t\right)\right]
$$

where

$$
E_{\text {out }}=-\frac{\mathrm{i} k \bar{h}}{2} \int_{0}^{a} \widehat{B}(z) \exp \left(\frac{\mathrm{i} \Delta k^{2} z}{2 k}\right) \mathrm{d} z
$$

and $k_{\text {out }}=k_{\text {loc }}(z=a) .{ }^{8}$ This shows that the effective size of the "gravitational EM wave transmitter" is determined by either the extension of the magnetic field, provided the direction of the static magnetic field does not oscillate, or the mismatch distance $L=2 k / \pi \Delta k^{2}$, whichever is smaller. This is a consequence of the fact that extra-ordinary mode and gravitational waves satisfy nearly the same dispersion relation in the regime of consideration, which tends to make linear wave interaction coherent over large distances.

\section{Applications to astrophysics}

It is an interesting question whether it is possible to detect the generated EM waves during realistic conditions. An immediate problem is that for most binary astrophysical sources, such

\footnotetext{
${ }^{7}$ Specifically this result holds in a vacuum region, and in this case the effect has been used as an argument for employing EM fields as gravitational wave detectors (see e.g. Grischuk \& Polnarev (1980) and references therein).

${ }^{8}$ If we insist on a sharply defined excitation region $0<z<a, k_{\text {out }}$ typically becomes imaginary, reflecting the fact that waves with $\omega<\omega_{\mathrm{p}}$ does not propagate in an unmagnetized one-component plasma.
} 
as binary neutron stars (Rasio \& Shapiro 1999), $\omega \lesssim 10^{3} \mathrm{~Hz}$, whereas the plasma density for typical interstellar matter correspond to $\omega_{\mathrm{p}} \gtrsim 10^{3} \mathrm{~Hz}$. Although an external magnetic field may decrease the cut-off frequency significantly below $\omega_{\mathrm{p}}$ for EM waves propagating perpendicular to the magnetic field, we cannot rely on that effect for EM wave propagation over interstellar distances. Furthermore, observation from the ground are inaccessible for frequencises roughly below $10 \mathrm{MHz}$, due to the ionospheric cut-off. However, as we will demonstrate below, it is too early to conclude that gravitationally induced EM waves cannot be detected.

Let us consider a binary system consisting of two identical pulsars,${ }^{9}$ with individual masses $M_{\odot}$ (for a general review of the physical properties of binary pulsars, see Phinney \& Kulkarni (1994)). This leads to a Schwarzschild radius $R_{\mathrm{S}}=2 G M_{\odot} / c^{2} \approx 3 \mathrm{~km}$. Furthermore, we assume that the separation distance between the two neutron stars is $d=20 R_{\mathrm{S}}$. Using a Newtonian approximation, we find that the gravitational wave frequency is $\omega \approx 2 \times 10^{3} \mathrm{rad} / \mathrm{s}$. The unperturbed magnetic field is taken as $B(r)=B_{\text {surf }} r_{\text {puls }}^{3} / r^{3}$, where the surface magnetic field $B_{\text {surf }}$ is of the order $10^{6}-10^{10} \mathrm{~T}$ (where the upper limit refers to magnetars), and $r_{\text {puls }}$ is the radius of the pulsar. Thus the fall-off of the magnetic field is that of a dipole.

Next we want to estimate the maximum electric field from equation (20a). To get a magnetic field that is essentially static in the frame of reference where the binary system is rotating, we must exclude the region closest to the pulsar surface from the region of interaction. Assuming that the electromagnetic excitation effectively starts at a distance $r=60 R_{\mathrm{S}}$ from the center of mass of the system, using the estimates presented above, together with condition (18), we find from equation (20a) that

$$
E_{\text {max }} \sim 1.5 \times 10^{6} \bar{h}_{\text {int }} B_{\text {surf }} \mathrm{V} / \mathrm{m}
$$

where we have denoted the gravitational wave amplitude at the beginning of the interaction region $\left(r=60 R_{\mathrm{S}}\right)$ by $\bar{h}_{\text {int }}$. Here we have used that the effective interaction distance is determined by a fall off in the interaction efficiency together with a spherical attenuation of the electromagnetic wave, rather than a decoherence of the modes as discussed in the analytical calculations. Using ${ }^{10}$ $\bar{h}_{\text {int }} \sim 0.001$ and $B_{\text {surf }} \sim 10^{6} \mathrm{~T}$, equation $(20 \mathrm{~b})$ gives

$$
E_{\max } \sim 500 \mathrm{kV} / \mathrm{m}
$$

at a distance $r_{\max } \approx 120 R_{\mathrm{S}}$. In this region, the induced electron velocity is kept non-relativistic to a good approximation - by the external magnetic field, since the electrical and magnetic fields

\footnotetext{
${ }^{9}$ One might be hesitant to apply our results derived for a one-component plasma, to the electron-positron plasma that exists in the vicinity of pulsars. For our case, however, the presence of the plasma is not very important in the EM excitation region, due to the strong magnetization which keeps down the mobility, but instead the plasma affects the EM wave propagation further away from the binary pulsar, as to be discussed below. For a discussion of EM excitations by gravitational waves in a multi-component plasma, see Ignat'ev (1997).

${ }^{10}$ Using a Newtonian approximation with $d=\alpha R_{\mathrm{S}}, r=\beta R_{\mathrm{S}}$, we find that $\bar{h} \sim(2 \alpha \beta)^{-1}$.
} 
are perpendicular, and we have $v \approx E_{\max } / B\left(r_{\max }\right) \approx 6 \times 10^{5} \mathrm{~m} / \mathrm{s}$. Using an $1 / r$ spherical decay of the EM field for $r>r_{\max }$, the electron velocity can also be estimated at large distances. In a distant region where the magnetic field is assumed to have decreased such that $\omega_{\mathrm{c}} \lesssim \omega$, the electron velocity is of the order $v(r) \sim q E_{\max } r_{\max } /(m \omega r)$, provided the ultrarelativistic regime is not reached. The radius $r_{\text {rel }}$ from the source where the electron quiver velocity is moderately relativistic (which we define to be $v=10^{8} \mathrm{~m} / \mathrm{s}$ ) can then be solved for. We obtain

$$
r_{\text {rel }} \sim 7.5 \times 10^{7} \mathrm{~km} \sim 5 \mathrm{au} .
$$

Since the magnetic field from the binary system is weak at this distance (i.e. the condition $\omega_{\mathrm{c}} \lesssim \omega$ is fulfilled), we deduce that the electron velocity must become relativistic at some distance $r$ satisfying $r_{\text {max }} \ll r \ll r_{\text {rel }}-$ the precise value depend on extension of the magnetic field. ${ }^{11}$

When the electron velocities approaches the speed of light the excited EM wave becomes highly nonlinear, as the ponderomotive and relativistic nonlinearities in the momentum equation becomes comparable to the linear terms (Shukla, Yu \& Tsintsadze 1986). Several nonlinear effects may take place, such as parametric excitation of other plasma waves (exciting lower frequencies) and harmonic generation of the original mode. A detailed study of the evolution of the EM waves is by far beyond the scope of our investigation. However, we note that the degree of inhomogeneity of the background medium may be crucial for the outcome. From now on we assume that the nonlinear regime is reached comparatively close to the binary source, where the plasma medium can be characterized as strongly imhomogeneous. This means that the background parameters $\omega_{\mathrm{c}}$ and $\omega_{\mathrm{p}}$ are assumed to change significantly during a single wavelength of the EM wave. Strong inhomogeneities generally tend to supress parametric excitations (Rosenbluth, White \& Liu 1973), and thus we expect harmonic generation to be the more important process in our case. To get a feeling for the possible magnitude of this effect, we can compare with laser experiments (Carman, Forslund, \& Kindel 1981; Carman, Rhodes, \& Benjamin 1981), where the relativistic velocities induced in an inhomogeneous plasma leads to repeated harmonic generation by a factor of order 50 of the original laser frequency, and these harmonics have a comparable amplitude to that of the original frequency (see Bezzerides, Jones, \& Forslund (1982) and Grebogin, Triphati, \& Chen (1983) for a theoretical explanation of this effect). For the parameters chosen in our example, the electron velocity can be as large as in the experiments presented by Carman, Forslund, \& Kindel (1981) and Carman, Rhodes, \& Benjamin (1981). The inhomogeneity parameter $1 / k L$, where $L$ is the inhomogeneity scale length, is also of comparable magnitude to the same experiments, and thus it is not unrealistic to assume that a significant part of the excited EM energy can be converted to higher harmonics with a harmonic number between 40 and 50 of the original frequency, which correspond to a EM wave frequency in the long wavelength radio regime, but well above the typical cut-off frequency of the interstellar plasma. Such EM waves can propagate over astrophysical distances and suffer no other significant damping than spherical attenuation.

\footnotetext{
${ }^{11}$ It should be noted that the $1 / r^{3}$ behaviour of the magnetic field holds to the light cylinder, outside of which the magnetic field falls off as $1 / r$ (Rees 1999).
} 
Detection of such low frequecy signals cannot be done on earth, due to absorption and reflection of the ionospheric plasma. Thus it is evident that no existing radio telescopes can measure the electromagnetic emissions discussed above, but well developed proposals like the Astronomical Low Frequency Array (ALFA) is expected to measure electromagnetic signals in the interval $30 \mathrm{kHz}$ to $30 \mathrm{Mhz}$ (Jones et al. 1998). Assuming that $10 \%$ of the energy gets transferred to harmonic numbers with a high enough frequency for detection (roughly harmonic number $n \gtrsim 60$ ), and estimating the minimum detection level (due to noise originating from the solar system) to 1000 Jy (Jones 1999), we deduce that the source considered above can be observed by a system such as ALFA provided it is located to our own galaxy. ${ }^{12}$

It is of interest to consider possible effects due to strong gravitational field deviations from Minkowski spacetime. According to Daniel \& Tajima (1997) all frequencies, e.g. the plasma- and cyclotron frequencies, are redshifted by the lapse function in a spherically symmetric spacetime. Since the important quantity in the above calculations are the quotient of certain frequencies, the effect will persist, if we assume that the present situation can be approximated as spherical symmetric. Of course, the frequency of the emitted radiation will be redshifted $\left(\omega_{\text {emmision }} / \omega_{\text {reception }}=1+z\right)$, although $z$ will be less than $2 \%$ for the interaction distance $60 R_{\mathrm{S}}$ used in the above example.

\section{Summary and discussion}

In this letter we have investigated the interactions between weak gravitational waves and EM fields, using the $1+3$ Orthonormal Frame formalism. In this way we were able to give a clear presentation of the result that an EM wave can be generated as a result of the interaction of a gravitational wave with a static isolated magnetic field. Furthermore, in the presence of a plasma we argued that higher harmonics of the original EM wave may be generated.

In light of these results we will discuss the possible cosmological implications in a forthcoming paper (Marklund, Dunsby, \& Brodin 1999), using the 1+3 Covariant approach and tetrad methods which in a clear unified way deals with such problems. For example, we speculate that this may lead to an imprint on the Cosmic Backround Radiation (CBR) spectrum through the interaction between primordial gravitational waves and magnetic fields of cosmological origin. Therefore CBR measurements could in principle be used to constrain cosmological magnetic fields.

M. M. was supported by the Royal Swedish Academy of Sciences. P. K. S. D. was supported by the NRC (South Africa) and the URC (University of Cape Town).

\footnotetext{
${ }^{12}$ Furthermore, we excpect (at least for neutron stars with stiff equations of state (Rasio \& Shapiro 1999)) that at the final stage of the inspiral process, the frequency and amplitude of the gravitational radiation will increase, making the process more effective over a short time-span
} 


\section{REFERENCES}

Bezzerides, B., Jones, R. D., \& Forslund, D. W. 1982, Phys. Rev. Lett., 49, 202

Braginskii, V. B., Grishchuk, L. P., Doroshkevich, A. G., Zel'dovich, Ya. B., Novikov, I. D., \& Sazhin, M. V. 1974, Sov. Phys.- JETP, 38, 865 ; 1973, Zh. Eksp. Teor. Fiz., 65, 1729

Carman, R. L., Forslund, D. W., \& Kindel, J. M. 1981, Phys. Rev. Lett., 46, 29

Carman, R. L., Rhodes, C. K., \& Benjamin, R. F. 1981, Phys. Rev. A, 24, 2649

Cooperstock, F. I. 1968, Ann. Phys., 47, 173

Daniel, J., \& Tajima, T. 1997, Phys. Rev. D, 55, 5193

Demiański, M. 1985, Relativistic Astrophysics, (Pergamon Press)

Denisov, V. I. 1978, Sov. Phys.- JETP, 42, 209; 1978, Zh. Eksp. Teor. Fiz., 74, 401

DeWitt, B. S., \& Brehme, R. W. 1960, Ann. Phys., 9, 220

Ellis, G. F. R., \& van Elst, H. 1998, Cosmological Models, in Cargése Summer School 1998 Proceedings, ed. Marc Lachieze-Rey, in press (http://xxx.lanl.gov/abs/gr-qc/9812046)

Gerlach, U. N. 1974, Phys. Rev. Lett., 32, 1023

Grebogi, C., Triphati, V. P., \& Chen, H-H. 1983, Phys. Fluids, 27, 1904

Grishchuk, L. P., \& Polnarev, A. G. 1980, in General Relativity and Gravitation, Vol. 2, ed. A. Held (Plenum Press)

Grishchuk, L. P., \& Sazhin, M. V. 1976, Sov. Phys.- JETP, 41, 787; 1975, Zh. Eksp. Teor. Fiz., 68, 1569

Ignat'ev, Yu. G. 1997 Phys. Lett. A., 230, 171

Jones, D. L. et al. 1998, Radio Emission from Galactic and Extragalactic Compact Sources, ASP Conference Series, Vol. 144, IAU Colloquium 164, eds. J. A. Zensus, G. B. Taylor, \& J. M. Wrobel, p. 393

Jones, D. L. 1999, private communication

Lupanov, G. A. 1967, Sov. Phys.- JETP, 25, 76; 1967, Zh. Eksp. Teor. Fiz., 52, 118

Macdonald, D., \& Thorne, K. S. 1982, MNRAS, 198, 345

Marklund, M., Dunsby, P. K. S., \& Brodin, G. 1999, in preparation

Misner, C., Thorne, K. S., \& Wheeler, J. A. 1973, Gravitation, (Freeman) 
Phinney, E. S., \& Kulkarni, S. R. 1994, ARA\&A, 32, 591

Rasio, F. A., \& Shapiro, S. L. 1999, Class. Quantum Grav., 16, R1

Rees, M. 1999, private communication

Rosenbluth, M. N., White, R. B., \& Liu, C. S. 1973, Phys. Rev. Lett., 31, 697

Shukla, P. K., Yu, M. Y., \& Tsintsadze, N. L. 1986, Phys. Rep., 138, 1

Stephani, H. 1982, General Relativity (Cambridge: Cambridge University Press)

Zeldovich, Ya. B. 1974, Sov. Phys.- JETP, 38, 652; 1973, Zh. Eksp. Teor. Fiz., 65, 1311 